\title{
Performance of nonsynchronous noncommensurate impedance transformers in comparison to tapered line transformers
}

Kim, Kseniya; Zhurbenko, Vitaliy; Johansen, Tom Keinicke; Narendra, Kumar

Published in:

Proceedings of European Microwave Conference 2012

Publication date:

2012

Document Version

Peer reviewed version

Link back to DTU Orbit

Citation (APA):

Kim, K., Zhurbenko, V., Johansen, T. K., \& Narendra, K. (2012). Performance of nonsynchronous noncommensurate impedance transformers in comparison to tapered line transformers. In Proceedings of European Microwave Conference 2012 http://www.eumweek.com/2012/EuMC.asp?id=c

\section{General rights}

Copyright and moral rights for the publications made accessible in the public portal are retained by the authors and/or other copyright owners and it is a condition of accessing publications that users recognise and abide by the legal requirements associated with these rights.

- Users may download and print one copy of any publication from the public portal for the purpose of private study or research.

- You may not further distribute the material or use it for any profit-making activity or commercial gain

- You may freely distribute the URL identifying the publication in the public portal 


\section{Performance of Nonsynchronous Noncommensurate Impedance Transformers in Comparison to Tapered Line Transformers}

\author{
Kseniya Kim, Vitaliy Zhurbenko, \\ Tom Keinicke Johansen \\ Technical University of Denmark \\ Kgs. Lyngby, Denmark \\ vz,tkj@elektro.dtu.dk
}

\author{
Kumar Narendra \\ Research \& Development Center, Motorola Technology \\ Penang, Malaysia \\ cnk020@motorola.com
}

\begin{abstract}
Nonsynchronous noncommensurate impedance transformers consist of a combination of high- and lowimpedance transmission lines. The present work is dedicated to the synthesis of such transformers. They are analyzed employing wave transmission matrices. The performance of the transformer is compared to a traditional tapered line impedance transformer.

The increase in bandwidth of nonsynchronous noncommensurate impedance transformers typically leads to shortening the transformer length, which makes the transformer attractive for applications, where a wide operating band and high transformation ratios are required.
\end{abstract}

Keywords- impedance matching, wave transmission matrices.

\section{INTRODUCTION}

The design of wideband impedance matching circuits is a challenging task, because the traditional designs based on distributed components exhibit a large circuit size which is highly undesirable in practice. The matching circuits based on lumped elements, however, are compact but suffer from low $Q$-factor at high frequencies.

Nonsynchronous noncommensurate altering transmission line impedance transformers (NN impedance transformers) are one of the promising types of transformers for a broadband matching. They provide a wide bandwidth, comparatively low insertion loss, have a compact size, and exhibit a comparatively low sensitivity to fabrication errors. Contrary to the transformers described in [1], NN impedance transformers consist of the sections of different lengths (noncommensurate) with the same characteristic impedances as the impedances of the source $\mathrm{Z}_{0}$ and the load $\mathrm{Z}_{\mathrm{L}}$ which should be matched. The word nonsynchronous in the name of the impedance transformer means that the impedance ratio between the steps can be equal or exceed the output-to-input transformation ratio $r$ by a large factor. In some literature $\mathrm{NN}$ impedance transformers are also referred as stepped transformers of Class II [2].

The work presented in this paper complements the design data presented in [3] and [4], and the synthesis procedure of a 12 -section $\mathrm{NN}$ impedance transformer with transformation ratios $r=2 \ldots 10$ and bandwidth ratios $\chi=4 \ldots 20$ is given here as an example. The performances of the synthesized NN transformer are then compared to those of a traditional tapered line impedance transformer.

\section{ANALYSIS BASED ON WAVE TRANSMISSION MATRICES}

For analysis of NN impedance transformers, the approach based on wave transmission matrices (T-matrices) has been employed, allowing to consider multiple reflections from the impedance transformer sections. NN impedance transformer is assumed to be lossless, reciprocal, and antimetric two-port network $\quad\left(\mathrm{T}_{11}=\mathrm{T}_{22}{ }^{*}, \quad\left|\mathrm{~T}_{11}\right|^{2}=1+\left|\mathrm{T}_{12}\right|^{2}, \quad \mathrm{~T}_{12}=\mathrm{T}_{21}\right.$, $\left.\operatorname{Im} \mathrm{T}_{12}=\operatorname{Im} \mathrm{T}_{21}=0\right)$. These assumptions simplify the analysis still providing acceptable results. The antimetry yields that the sections' lengths $\theta_{\mathrm{n}}$ are symmetric with regard to the transformer center $\theta_{n}=\theta_{N-(n-1)}, \quad n=1 \div N / 2$, and the partial reflection coefficients from the steps discontinuities $\rho_{\mathrm{n}}$ are also symmetric with regard to the transformer centre $\rho_{\mathrm{n}-1}=\rho_{\mathrm{N}-(\mathrm{n}-1)}$, $n=1 \div N / 2$, where $N$ is the number of sections. Moreover, the sections' electrical lengths are related to each other linearly $\theta_{\mathrm{n}}=v_{\mathrm{n}} \theta_{1}, n=2 \div N$, where $\theta_{1}$ is the electrical length of the first section at the center frequency $f_{0}$ of the matching bandwidth, $v_{\mathrm{n}}$ is the $n$-th component of the $N$-dimensional vector defining the electrical lengths of the remaining sections $v=\left(1, v_{2}, v_{3}, \ldots\right.$, $v_{\mathrm{N}}$ ).

A schematic view of a 12-section $\mathrm{NN}$ impedance transformer is shown in Fig.1.

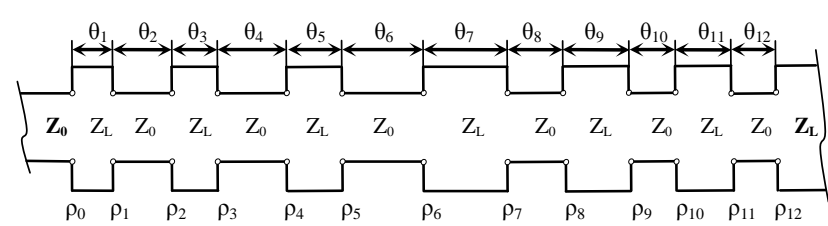

Fig.1. A schematic view of a 12 -section $\mathrm{NN}$ impedance transformer

The transformer consists of altering sections with the electrical lengths $\theta_{1}=\theta_{12}, \theta_{2}=\theta_{11}, \theta_{3}=\theta_{10}, \theta_{4}=\theta_{9}, \theta_{5}=\theta_{8}, \theta_{6}=\theta_{7}$ and having the same characteristic impedances as the impedance of the source $Z_{0}$ and the load $Z_{L}$. The model of NN impedance transformer consists of two-port networks formed by two transmission lines with different characteristic 
impedances, and the entire impedance transformer is treated as series connected two-port networks.

In a two-port network, the directions of wave propagation have been chosen so that $\mathrm{V}_{1}^{+}, \mathrm{V}_{2}^{-}$are the voltage amplitudes of the waves entering port 1 and port 2 , and $\mathrm{V}_{1}^{-}, \mathrm{V}_{2}{ }^{+}$are the voltage amplitudes of outgoing waves from port 1 and port 2 , respectively.

For the chosen directions of propagation, the scattering transfer matrix can be written as

$$
\left[\begin{array}{l}
V_{1}^{+} \\
V_{1}^{-}
\end{array}\right]=\left[\begin{array}{ll}
T_{11} & T_{12} \\
T_{21} & T_{22}
\end{array}\right]\left[\begin{array}{l}
V_{2}^{+} \\
V_{2}^{-}
\end{array}\right]
$$

The first two-port network of NN impedance transformer is formed by shifting the reference plane of the impedance transformer for a half of electrical length $\theta_{1} / 2$ in the direction of the source. All the following two-port networks are respectively consist of two transmission lines with length $\theta_{1} / 2$ and $\theta_{n}-\theta_{1} / 2, n=2 \div N$. For a two-port network between $n$ and $(n+1)$ step, formed by two transmission lines with different characteristic impedances, T-matrix can be expressed as

$$
[T]_{n}=\frac{1}{\sqrt{1-\rho_{n}^{2}}}\left[\begin{array}{cc}
e^{j \theta_{n+1}} & \rho_{n} e^{-j\left(\theta_{n+1}-\theta_{1}\right)} \\
\rho_{n} e^{j\left(\theta_{n+1}-\theta_{1}\right)} & e^{-j \theta_{n+1}}
\end{array}\right]
$$

The impedance transformer has more section discontinuities $(N+1)$ than the number of sections $(N)$, therefore, the last twoport network is formed by combining two transmission lines of length $\theta_{1} / 2$.

The resulting T-matrix of the 12-section NN impedance transformer is found by a consecutive $T$-matrices multiplication for the impedance transformer sections

$$
[T]_{\text {oot }}=[T]_{1} \cdot[T]_{2} \cdot \ldots \cdot[T]_{12}
$$

The parameters of the NN impedance transformer are found solving a mini-max problem for the magnitude of the total reflection coefficient

$$
\operatorname{Min}|\Gamma|_{\max },|\Gamma|_{\max }=\max \left|\Gamma\left(\theta_{1}, v\right)\right|, \theta_{1} \in\left[\theta_{1}\left(f_{1}\right), \theta_{1}\left(f_{2}\right)\right]
$$

where $\theta_{1}\left(f_{1}\right)$ and $\theta_{1}\left(f_{2}\right)$ are the electrical lengths of the first section $\theta_{1}$ at the lowest frequency $f_{1}$ and the highest frequency $f_{2}$ of the assumed matching bandwidth and

$$
|\Gamma|_{\max }=\frac{\left|T_{21 \text { tot }}\right|_{\max }}{\sqrt{1+\left|T_{21 \text { tot }}\right|_{\max }^{2}}}
$$

where $T_{21 \text { tot }}$ is the scattering transfer coefficient of the overall $\mathrm{NN}$ impedance transformer.

\section{DESIGN DATA FOR A SYNTHESIS OF 12-SECTION NN IMPEDANCE TRANSFORMER}

Figures 2-9 represent the design data for the synthesis of 12-section NN impedance transformer with bandwidth ratios $r=2 \ldots 10$, the transformation ratios $\chi=4 \ldots 20$, and the maximum reflection coefficient magnitude $|\Gamma| \leq-10 \mathrm{~dB}$. The data have been obtained by solving (4) numerically.

The given range of bandwidth ratios and transformation ratios has been chosen for practical reasons. $\chi>20$ and $r>10$ would lead to poor matching. In most practical cases, this would require implementation of a larger transformer with number of sections of more than twelve. Choosing $\chi<4$ would lead to a very low level of reflection coefficient and in most practical cases it would be reasonable to use a shorter transformer (with a number of sections less than twelve).

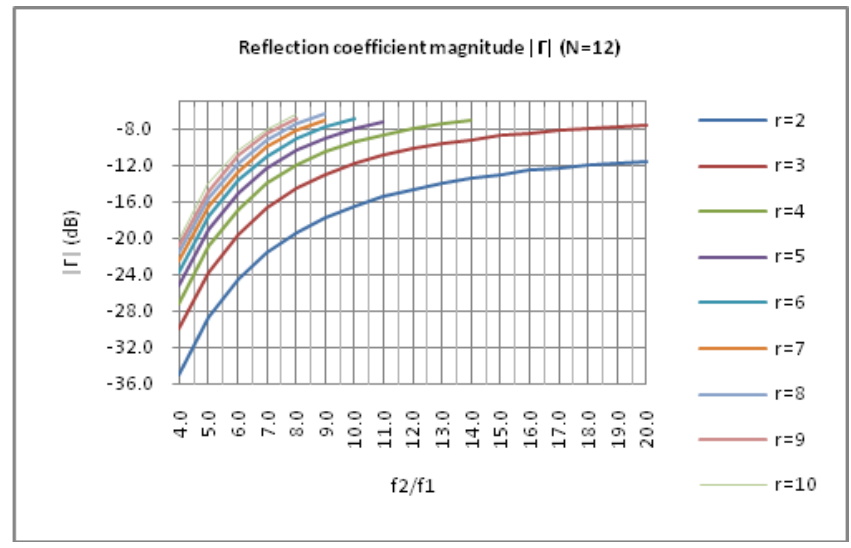

Fig.2. The magnitude of the reflection coefficient for the 12-section NN impedance transformer.

The magnitude of the reflection coefficient, $|\Gamma|$, of a 12section NN impedance transformer can be found from Fig.2. $|\Gamma|$ deteriorates with increasing transformation ratio $r$ and bandwidth ratio $\chi$. Fig.3-8 show the sections' electrical lengths.

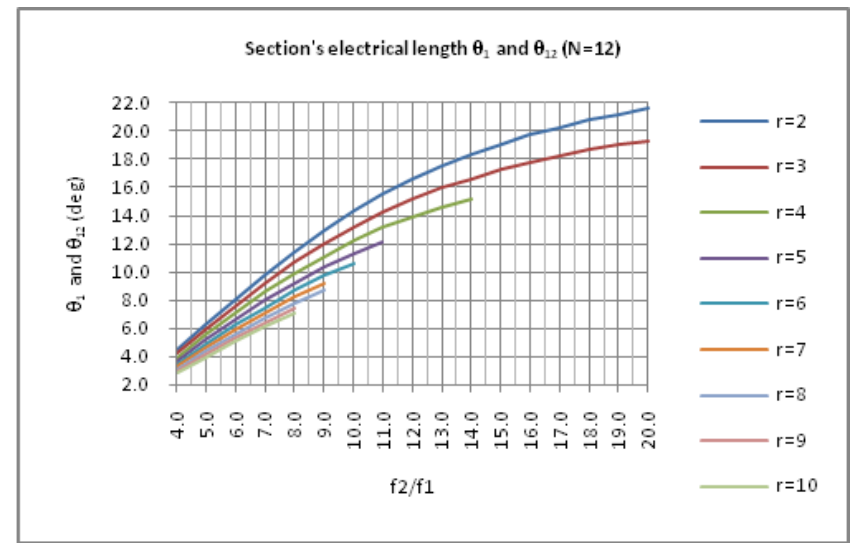

Fig.3. The electrical length of the sections 1 and 12 for the transformer in Fig. 1.

The electrical length of the first and twelfth sections, third and tenth sections, and the fifth and eighth sections of the 12section NN impedance transformer is increasing while 
increasing the bandwidth ratio and decreasing the transformation ratio.

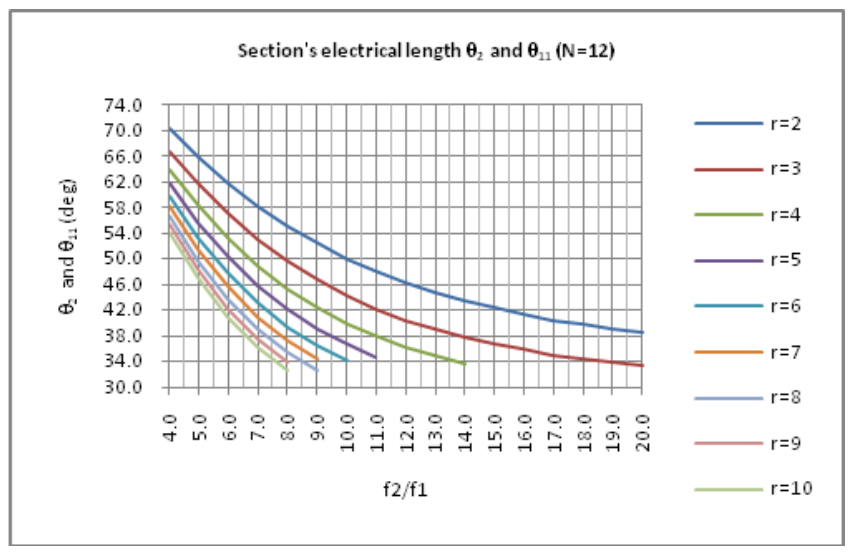

Fig.4. The electrical length of the sections 2 and 11 for the transformer in Fig. 1.

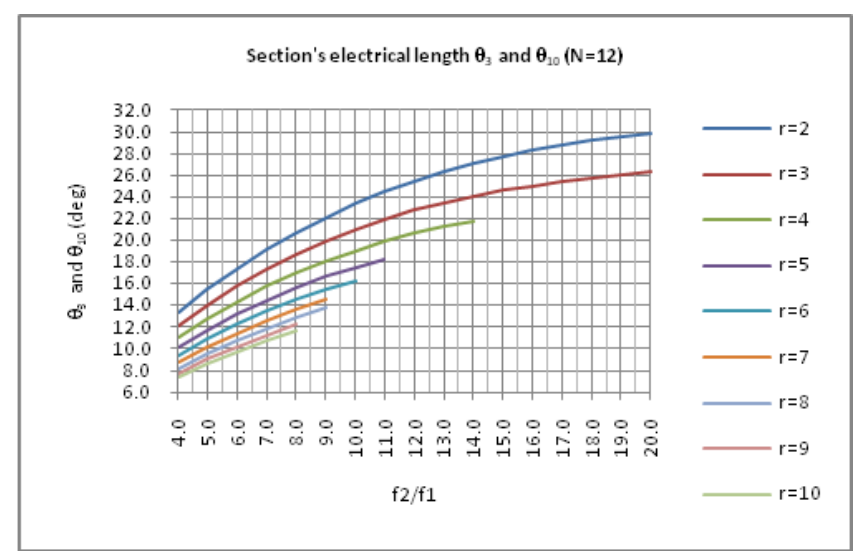

Fig.5. The electrical length of the sections 3 and 10 for the transformer in Fig. 1.

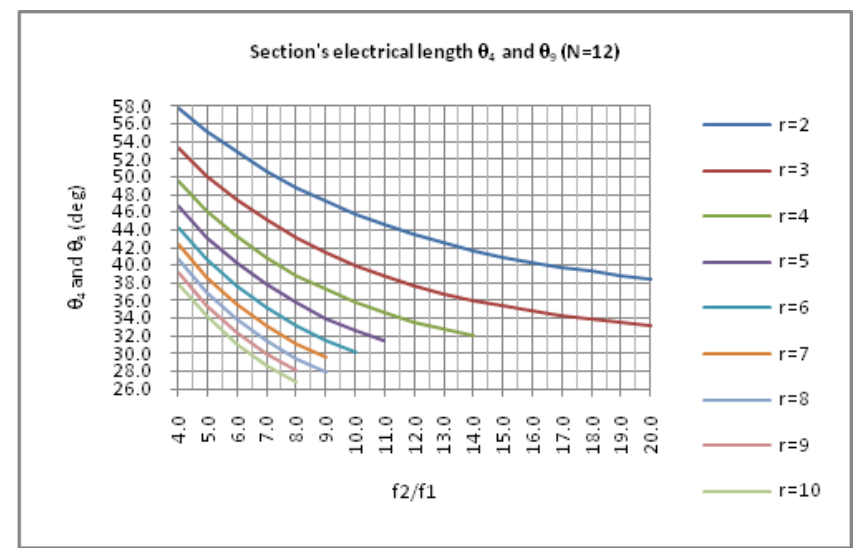

Fig.6. The electrical length of the sections 4 and 9 for the transformer in Fig. 1.

The electrical length of the second and eleventh sections, fourth and ninth sections, and sixth and seventh sections of the 12-section NN impedance transformer is decreasing while increasing the bandwidth ratio and the transformation ratio.

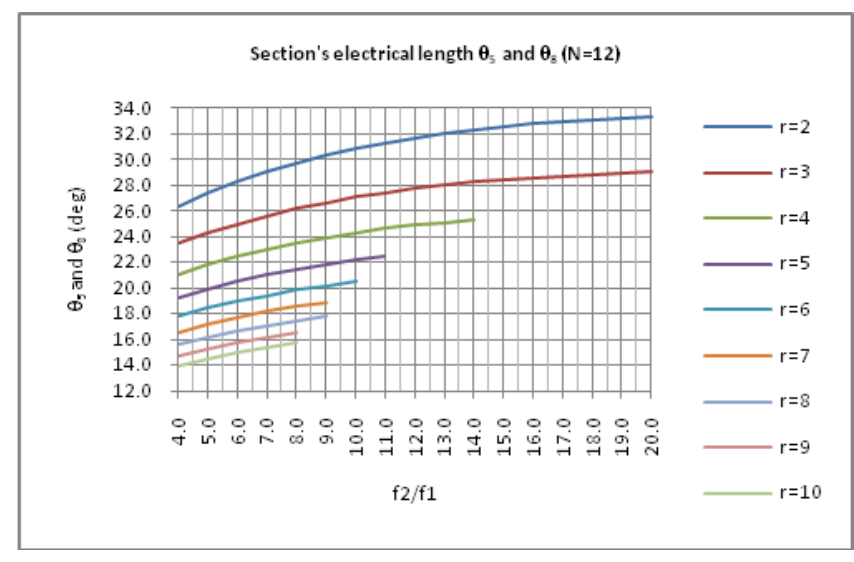

Fig.7. The electrical length of the sections 5 and 8 for the transformer in Fig. 1.

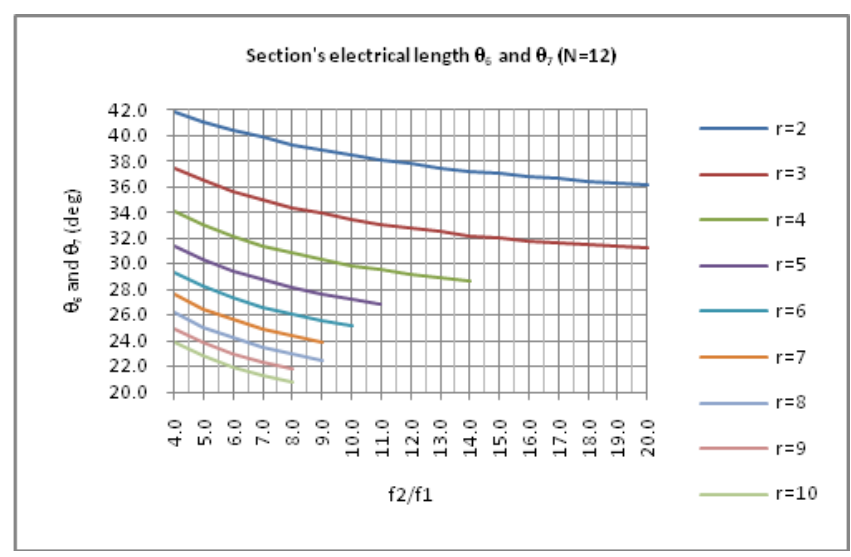

Fig.8. The electrical length of the sections 6 and 7 for the transformer in Fig. 1.

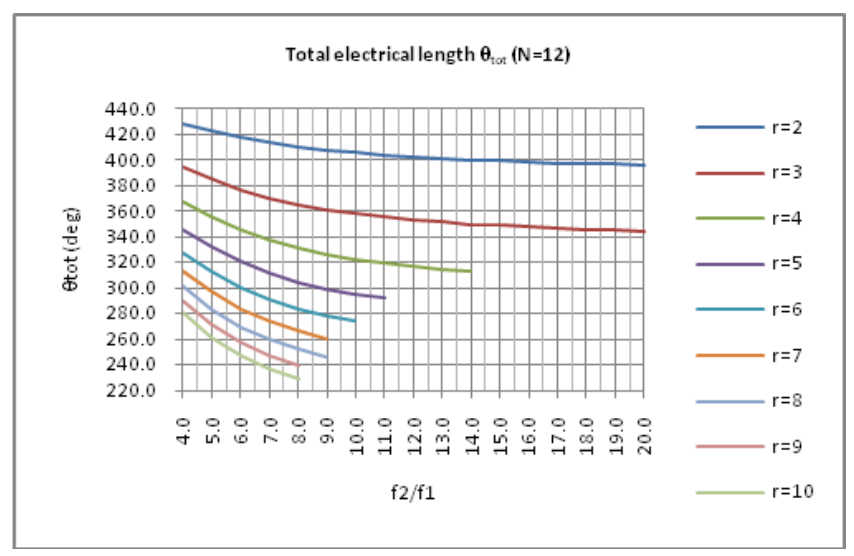

Fig.9. The total electrical length of a 12-section NN impedance transformer.

The total electrical length of the impedance transformer becomes shorter while increasing the bandwidth ratio $\chi$ and the transformation ratio $r$, as shown in Fig. 9.

IV. COMPARISON OF THE 12-SECTION NN IMPEDANCE TRANSFORMER WITH A TAPERED IMPEDANCE TRANSFORMER In order to validate the data obtained for the design of NN 
impedance transformers, an impedance transformer with a transformation ratio $r=4 \quad\left(\mathrm{Z}_{\mathrm{L}}=50 \quad \mathrm{Ohm}, \mathrm{Z}_{0}=12.5 \mathrm{Ohm}\right)$, bandwidth ratio $\chi=5\left(\mathrm{f}_{2}=2.25 \mathrm{GHz}, \mathrm{f}_{1}=0.45 \mathrm{GHz}\right)$, and the magnitude of the reflection coefficient better than $|\Gamma| \leq-20 \mathrm{~dB}$ has been synthesized based on the design data in Fig.3-8. The calculated $\mathrm{S}$-parameters of the impedance transformer using equation (5) are shown in Fig. 10.

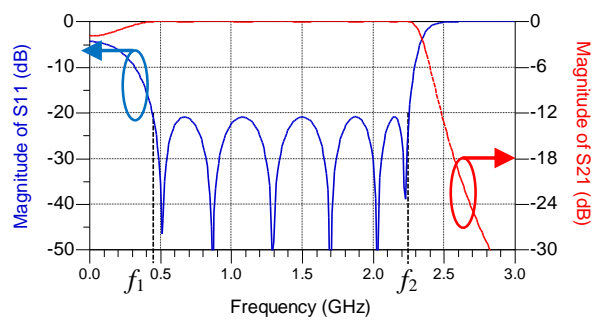

Fig.10. Magnitudes of $S_{11}$ and $S_{21}$ of the 12-section NN impedance transformer.

To compare the performance of a 12 -section NN impedance transformer with a tapered impedance transformer, they have been realized using RO4003 substrate with a thickness $h=1.524 \mathrm{~mm}$, relative dielectric constant $\varepsilon_{\mathrm{r}}=3.55$, dielectric loss tangent $\tan \delta=0.0021$, and conductor thickness $t=0.035 \mathrm{~mm}$. The length of the tapered transformer has been chosen such that it has the same frequency of the reflection coefficient first minimum as an $\mathrm{NN}$ impedance transformer.

The photo of the fabricated transformers is shown in Fig.11.

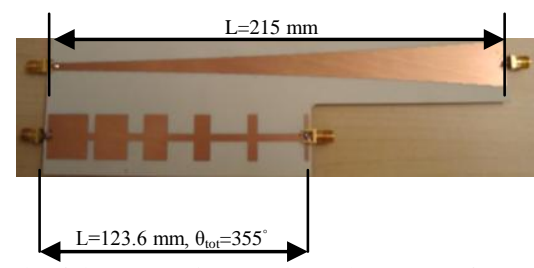

Fig.11. Photograph of the 12-section NN impedance transformer and tapered transformer manufactured on RO4003.

The impedance transformers have been measured using a vector network analyzer. The actual scattering parameters of the impedance transformer have been obtained through the renormalization and de-embedding.

Simulated and measured S-parameters of the impedance transformers are shown in Fig. 12.

Measurements show that the magnitude of the reflection coefficient of NN impedance transformer deteriorates at higher frequencies. One minimum is not visible compared to the MoM simulations. This is most probably caused by the inaccuracy of the fixture model in the de-embedding procedure. The simulated and measured reflection coefficients of the tapered impedance transformer also differ due to tolerance in fabrication and discontinuity of $12.5 \mathrm{Ohm}$-to$50 \mathrm{Ohm}$ transmission line step at $12.5 \mathrm{Ohm}$ port. The reflection coefficient magnitude doesn't decrease (improves) continuously while frequency increases, as it was expected.
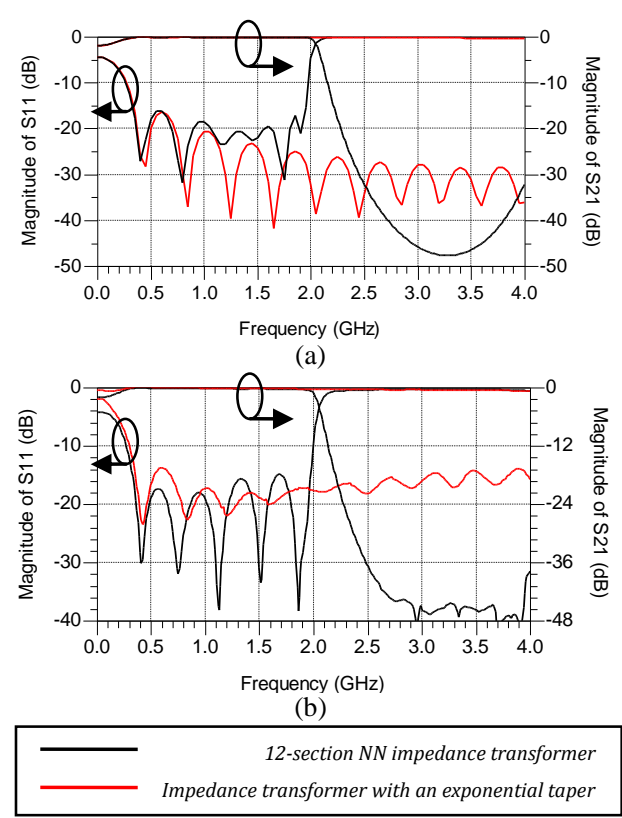

Fig.12 S-parameters of the NN impedance transformers in Fig. 11. (a) MoM simulations. (b) Measurements.

It can be concluded that the NN impedance transformer is more attractive in practice since it provides approximately the same matching level as the tapered impedance transformer in the specified frequency range, but has shorter length. A further miniaturization can be achieved by meandering the high impedance transmission line sections of the transformer [5].

\section{CONCLUSIONS}

The advantage of NN impedance transformers is that they generally exhibit considerably shorter length than the tapered impedance transformers in a fixed frequency range. It was also found, that the fabrication accuracy of tapered transformer should be high in order to achieve theoretically predicted continuous increase of reflection loss. In practice, however, the reflection coefficient of tapered impedance transformer degrades with frequency. Due to the lowpass behavior, the NN impedance transformers are preferable for matching of power amplifiers, since the higher harmonics exhibited by the amplifier will be attenuated.

\section{REFERENCES}

[1] G.L. Matthaei, Short-Step Chebyshev Impedance Transformers, IEEE Trans. Microwave Theory Tech., vol. MTT-14, no. 8, pp.372-383, 1966.

[2] V.P. Meschanov, I. A. Rasukova, V. D. Tupikin, Stepped Transformers on TEM-Transmission Lines, IEEE Trans. on Microw. Theory and Tech., vol. 44, no. 6, pp. 793-798, June 1996.

[3] S. Rosloniec, Algorithms for the Computer-Aided Design of Nonsynchronous, Noncommensurate Transmission-Line Impedance Transformers, Int. Journal Microw. and Millimeter-Wave ComputerAided Engineering, vol. 4, no. 3. pp. 307-314, March 1994.

[4] C. M. Tsai, C. C. Tsai, and S. Y. Lee, Nonsynchronous AlteringImpedance Transformers, Asia-Pacific Microw. Conf., vol. 1, pp. 310313, December 2001.

[5] V. Zhurbenko, K. Kim, K. Narenda, A Compact Broadband Nonsynchronous Noncommensurate Impedance Transformer, Microw. and Optical Tech. Letters Vol. 54, No. 8, August 2012, pp. 1832-1835. 